\title{
Duale Studiengänge: Konkurrenz für die klassische Ausbildung?
}

\author{
WINFRIED HEIDEMANN, JOHANNES KOCH
}

Unternehmen bieten neben der dualen Ausbildung nach Berufsbildungsgesetz (BBiG) zunehmend auch Ausbildung in dualen Studiengängen an, in denen ein Hochschulstudium mit Ausbildung oder praktischer Tätigkeit im Betrieb verbunden wird. Als Vorteile werden Praxisnähe, kurze Studiendauer, gute Studienbedingungen, Arbeitsmarkt- und Karrierechancen genannt. ${ }^{\bullet}$ Auch als Strategie zur Bewältigung steigender Qualifikationsanforderungen und zur Erweiterung von Bildungschancen werden sie empfohlen. ${ }^{2}$ Die quantitative Entwicklung dieser Studiengänge ist relativ gut belegt. ${ }^{3}$ Demgegenüber sind die betrieblich-personalpolitischen und die qualifikationspolitischen Hintergründe empirisch kaum aufgehellt: Zeichnen sich hier neue Muster der betrieblichen Personalrekrutierung ab? Verdrängt diese Ausbildungsform die klassische Lehrlingsausbildung? Ist die Entwicklung zur Wissensgesellschaft ein Auslöser? Ergeben sich neue Bildungschancen für die Teilnehmer? Vor dem Hintergrund dieser Fragestellungen haben wir im Beratungskontext der Mitbestimmungsförderung der HansBöckler-Stiftung betriebliche Kurzfallstudien erstellt. Sie geben eine Reihe von aufschlussreichen Hinweisen, aber noch keine empirisch abgesicherten Antworten.

\section{Merkmale und Verbreitung dualer Studiengänge}

Duale Studiengänge nutzen zwei Lernorte in Hochschule und Wirtschaftsbetrieb oder Verwaltung. Es lassen sich vier Typen unterscheiden. ${ }^{\circ}$

(1) Ausbildungsintegrierende duale Studiengänge bieten ein Hochschulstudium mit einer parallelen Ausbildung in einem Ausbildungsberuf nach Berufsbildungsgesetz. Hier kommt die Berufsschule als dritter Lernort hinzu.

(2) Praxisintegrierende duale Studiengänge verbinden ein Studium mit längeren Praxisphasen im Betrieb ohne Abschluss in einem anerkannten Ausbildungsberuf.

(3) Berufsintegrierende duale Studiengänge kombinieren berufliche Praxis als Teilzeittätigkeit von Berufstätigen mit einem Studium.

(4) Studien, die Beschäftigte neben ihrer betrieblichen Volloder Teilzeittätigkeit absolvieren, werden auch als berufs- begleitende duale Studiengänge bezeichnet, obwohl es keine Einbeziehung der betrieblichen Praxis in die Ausbildung gibt.

Duale Studiengänge haben in den letzten Jahren einen erheblichen Aufschwung genommen. 2011 gab es den Angaben des Bundesinstituts für Berufsbildung (BIBB) zufolge 61.195 Studierende in 929 dualen Studiengängen. Gegenüber 2008 war das eine Steigerung um $35 \%$ bei den Studiengängen und fast $40 \%$ bei den Studierenden. Die Zahl der beteiligten Betriebe wird nicht erhoben.

Fast 90 \% der Studiengänge gehören zu den ausbildungsoder praxisintegrierenden Angeboten der beruflichen Erstausbildung. Knapp drei Fünftel sind an Fachhochschulen angesiedelt, es folgen Berufsakademien einschließlich der dualen Fachhochschule Baden-Württemberg mit mehr als einem Drittel der Angebote. Universitäten und „sonstige Hochschulen“ fallen mit $3 \%$ bzw. $2 \%$ der Angebote kaum ins Gewicht. Fast alle angebotenen Abschlüsse bieten den Bachelor-Abschluss, nur 2,4 \% schließen mit dem Master ab.

Es dominieren Angebote in den Wirtschaftswissenschaften mit mehr als der Hälfte der Studierenden, gefolgt von Technik- und Ingenieurwissenschaften, der Informatik und mit weitem Abstand dem Sozialwesen. Die beteiligten Unternehmen gehören einem breiten Spektrum an: Metall- und Elektroindustrie, Chemische Industrie, Banken und Versicherungen, Einzelhandel und Informationstechnologie.

Die Datenbank des deutschen Akkreditierungsrates weist im Jahr 2012439 akkreditierte duale Studiengänge

So das Bundesinstitut für Berufsbildung: http://www ausbildungplus.de/html/33.php (letzter Zugriff 05.09.2012).

(2) Berthold, C./Leichsenring, H./Kirst, S./Voegelin, L. (2010): Demographischer Wandel und Hochschulen - Der Ausbau des Dualen Studiums als Antwort auf den Fachkräftemangel, Gütersloh.

(3) Übersichten des Bundesinstituts für Berufsbildung (BIBB), unter http://www.ausbildungplus.de/html/30.php (letzter Zugriff: 05.09.2012).

(4) Kupfer, F./Mucke, K. (2010): Duale Studiengänge an Fachhochschulen nach der Umstellung auf Bachelorabschlüsse, Bonn. 
aus, also weniger als die Hälfte der vom BIBB genannten 929. ${ }^{\circ}$

Die Zahlen dürfen nicht darüber hinwegtäuschen, dass duale Studiengänge nur einen sehr kleinen Raum in der Ausbildungslandschaft einnehmen: Der Anteil dual Studierender macht unter $4 \%$ der Gesamtzahl der Studierenden und knapp 3 \% aller Auszubildenden im dualen System aus.

\section{Betriebsfallstudien}

Unsere Kurzfallstudien entstanden zwischen 2006 und 2011 in Großunternehmen mit Betriebsrat: fünf in der Chemischen Industrie, je eines im Telekommunikationsbereich und der Fahrzeugindustrie. ${ }^{\ominus}$ Auslöser der Recherchen waren jeweils Beratungen mit Betriebsräten für ihre Mitbestimmungsaufgaben, die Fragestellungen sind also beratungsorientiert. Die Studien geben Auskunft über die personalstrategischen und mitbestimmungspolitischen Zusammenhänge zunächst in diesen Unternehmen. Unsere Beratungserfahrungen geben aber Anlass zu der Annahme, dass die Ergebnisse durchaus typisch für Unternehmen dieser Größenordnung und Unternehmenskultur sind. Inwieweit sie verallgemeinert werden können, muss aber weiteren Untersuchungen vorbehalten bleiben.

Die Erhebungen wurden mit leitfadengestützten Informationsgesprächen mit Mitgliedern des Ausbildungs- oder Personalmanagements, Betriebsräten und Jugend- und Auszubildendenvertretungen sowie mit Teilnehmern an dualen Studiengängen durchgeführt. Zusätzlich wurden Verantwortliche der kooperierenden Hochschulen über ihre Erfahrungen befragt.

\section{Betriebliche Regelungen}

In vier der sieben Fälle handelt es sich um ausbildungsintegrierende Studiengänge, bei denen eine reguläre Ausbildung in einem Ausbildungsberuf mit einem Bachelor-Studiengang an einer privaten oder öffentlichen Fachhochschule verbunden wird. Zwei weitere Unternehmen organisieren die Ausbildung zwar nicht im Rahmen eines eingetragenen Ausbildungsverhältnisses, erreichen aber den Berufsabschluss über externe Zulassungen zur Abschlussprüfung. Ein Unternehmen verzichtet auf einen Abschluss nach BBiG, führt also die praxisintegrierende Variante durch. In einem Fall werden zusätzlich berufsintegrierende Studiengänge für Berufstätige nach einer abgeschlossenen beruflichen Ausbildung angeboten.

Zugangsvoraussetzung ist immer die allgemeine oder fachgebundene Hochschulreife; die Regelungen zum Hochschulzugang für Berufstätige ohne Abitur nach der Rahmenvereinbarung der Kultusministerkonferenz werden nicht angewendet. Bewerbungen erfolgen bei den Unternehmen, das betrieblich festgelegte Auswahlverfahren entscheidet über die Aufnahme in die Ausbildung. Einen Sonderfall stellt ein Unternehmen dar, in dem die Teilnahme im Jahre 2010 auf ein Stipendiensystem umgestellt wurde: Auszubildende können sich nach einem halben Jahr Ausbildungszeit für ein mit einem Firmenstipendium unterstütztes, ausbildungsbegleitendes Studium bewerben; dieser Weg soll später auch Beschäftigten mit abgeschlossener Ausbildung offen stehen, sodass es sich dann um einen berufsintegrierenden oder berufsbegleitenden Studiengang handelt.

Die Unternehmen bieten Ausbildung in naturwissenschaftlichen, Metall-/Elektro-, informationstechnischen und kaufmännischen Berufen an. Dazu gehören Abschlüsse des Bachelor of Engineering (Chemie, Metall, Elektro), Bachelor of Science (Wirtschaftsinformatik, Betriebswirtschaft) und Bachelor of Arts (Betriebswirtschaft).

Die Studiengänge sind an öffentlichen und bei zwei Unternehmen an privaten Fachhochschulen angesiedelt. Alle sind nach Hochschulrecht akkreditiert, die betriebliche Praxis spielt für die Vergabe von Leistungspunkten aber keine Rolle.

Bei der Ausbildung nach Berufsbildungsgesetz wird die Kammerprüfung unter Nutzung der Verkürzung der Ausbildungszeit für Bewerber mit Hochschulreife nach zwei Jahren abgelegt. Für das Studium werden als Regelzeit drei bis vier Jahre vorgegeben. In fünf Unternehmen besuchen die Teilnehmer neben der Hochschule auch die Berufsschule (obwohl sie dazu wegen Überschreitens der Altersgrenze nicht verpflichtet sind).

\section{Arbeits- und Lernbedingungen}

Für die Zusammenarbeit mit den Hochschulen haben die Unternehmen unterschiedliche Formen entwickelt. In zwei Fällen nutzen die Studierenden das Regelangebot der Hochschule und sind während der Semesterferien im Betrieb. Vier Betriebe haben mit den Hochschulen eigene Maßnahmen an bestimmten Wochentagen (auch samstags) vereinbart. Ein Unternehmen kombiniert für das Studium Teleteaching mit kurzen Präsenzphasen in eigenen Bildungszentren.

Der Status der Teilnehmer ist während der Ausbildung nach BBiG der von Auszubildenden, danach der von Trainees oder Praktikanten. In allen Fällen wird eine Ausbildungs- oder Praktikantenvergütung gezahlt, die zumeist etwas über den Vergütungen der „grundständigen“ Auszubildenden liegt. Soweit Studiengebühren erhoben werden, trägt das Unternehmen zumindest einen Teil davon.

Die Arbeitszeit im Betrieb richtet sich nach der der übrigen Auszubildenden, hinzu kommt die Zeit für das Studium, zum Teil abends oder am Wochenende.

(5) http://www.hs-kompass2.de/kompass/servlet/ SuperXmITabelle (letzter Zugriff: 10.09.2012).

6 Ausführliche Darstellungen in Heidemann, W. (2011): Duale Studiengänge in Unternehmen. Sieben Praxisbeispiele, Hans-Böckler-Stiftung, Arbeitspapier, Bildung und Qualifizierung (236), Düsseldorf. 
Die Übernahme nach erfolgreichem Studienabschluss ist unterschiedlich geregelt: In vier Unternehmen werden alle Absolventen mit einem vorher festgelegten Leistungsniveau übernommen, in den anderen drei Unternehmen war die Übernahme bisher auch der Regelfall, allerdings gab es hier keine entsprechenden formellen Vereinbarungen. Die dann anschließende Vergütung richtet sich immer nach der übernommenen Tätigkeit.

Die befragten Studierenden sind mit der Ausbildung trotz der hohen Zeitbelastung - sehr zufrieden. Dafür spielt neben der Ausbildungsvergütung die Erwartung des Aufstiegs in betriebliche Führungspositionen eine Rolle.

\section{Betriebliche Mitbestimmung}

Der Betriebsrat ist in allen Unternehmen an der Auswahl der Bewerber beteiligt. Überall sind die Teilnehmer bei den Wahlen zur Jugend- und Auszubildendenvertretung wahlberechtigt. Im Engagement der Betriebsräte liegt diese Ausbildungsform im Vergleich zur „grundständigen“ Ausbildung nach BBiG aber eher am Rande der Aufmerksamkeit. In drei Unternehmen gibt es Betriebsvereinbarungen, in zweien einen Tarifvertrag zu den Rahmenbedingungen. Die Inhalte der Ausbildung oder Praxis sind nicht Gegenstand der Vereinbarungen.

\section{Ausbildungspolitische Hintergründe}

Duale Studiengänge stehen im Kontext der Entwicklung von betrieblichen Qualifikationsbedarfen und personalund ausbildungspolitischen Handlungsmöglichkeiten der Betriebe. Umbrüche in der betrieblichen Ausbildungspraxis sehen wir nicht nur durch technische Änderungen, sondern auch durch Geschäftspolitiken, Organisationsentwicklung und Strategien von Personalbeschaffung und -entwicklung verursacht. Automatisierungsprozesse führen insbesondere durch die Nutzung der Möglichkeiten von Datennetzen zu neuen (steigenden) Anforderungen. Auf der Angebotsseite der Arbeitskräfte stehen die Folgen des demografischen Wandels und der Qualifikationsstruktur der Bewerber. Betriebe versuchen, ihren je spezifischen Personalbedarf (oftmals nur in Kurzfristbetrachtung) durch betriebsspezifische Lösungen zu befriedigen. Die neueren flexiblen Ausbildungsordnungen und das steigende Lebensalter der Bewerber eröffnen ihnen dafür Möglichkeiten.

Die Berufsbildungspolitik hat auf solche Veränderungen mit „prozessorientierten“ Ausbildungsordnungen reagiert. Diese geben den Betrieben nicht mehr bestimmte Techniken und Verfahren als verbindliche Ausbildungsinhalte vor, sondern beschreiben betriebliche Leistungsprozesse, in denen die Ausbildung durchgeführt werden soll. Dadurch können die Betriebe die Ausbildung besser an ihre betriebsspezifischen Anforderungen und an den technischen und organisatorischen Wandel anpassen. Diese Ausbildungsordnungen entsprechen auch der Entwicklung, dass Betriebe mit der Automatisierung Arbeit nicht mehr als einzelne Tätigkeiten, sondern als umfassende Prozesse mit möglichst wenig Schnittstellen organisieren. Als Folge werden von den Fachkräften nun auch Aufgaben der Organisation und Disposition verlangt, die bisher eher im Zuständigkeitsbereich von Führungskräften gelegen haben.

Vor diesem Hintergrund liegt die Befürchtung nahe, Betriebe könnten zulasten der Ausbildung nach Berufsbildungsgesetz auf Bachelorabsolventen zurückgreifen, um ihren Bedarf an erweiterten Qualifikationen - Überblickswissen, Zusammenhangswissen, Allgemeinkompetenzen zu decken. ${ }^{\circ}$

Unsere Fallstudien bestätigen diese Befürchtung nicht. In keinem Fall haben wir Hinweise gefunden, dass die Absolventen dualer Studiengänge anstelle der Fachkräfte der dualen Ausbildung eingesetzt werden sollen. Den steigenden Qualifikationsbedarf wollen alle Unternehmen auch zukünftig mit dieser Ausbildungsform decken, allerdings mit steigenden Anforderungen an die Bewerber. Unsere Fallstudien zeigen aber, dass die Unternehmen mit dualen Studiengängen einen Bedarf an Führungskräften auf unteren und mittleren Ebenen decken wollen, der durch die alte Verbindung von Ausbildung und anschließender Fortbildung nicht mehr ausreichend realisierbar scheint. Dahinter steht ein bisher wenig beachteter Zusammenhang zur dualen Ausbildung: Diese konnte in der Vergangenheit wegen der Selektivität im allgemeinen Bildungswesen auf ein großes Potenzial leistungsfähiger Bewerber zurückgreifen, aus denen die Betriebe ihre unteren und mittleren Führungskräfte aufgebaut und rekrutiert haben. Mit der Bildungsexpansion wird den Betrieben dieses Potenzial teilweise entzogen. Zwar können Betriebe auch Abiturienten für eine duale Ausbildung gewinnen, viele von ihnen nehmen jedoch anschließend ein Studium auf und sind damit für den Betrieb (zunächst) verloren. Unter diesem Aspekt bringen duale Studienangebote den Betrieben zwei Vorteile: Zum einen werden besonders leistungsfähige und -bereite Bewerber erreicht, damit wird eine Bestenauslese durchgeführt; zum anderen sichern sie eine Personalbindung - die Mehrzahl der Absolventen verbleibt im Unternehmen. Eine Konkurrenz zur dualen Ausbildung entsteht erst, wenn deren Absolventen nach einer Weiterbildung etwa zum Meister in die untere Führungsebene aufsteigen wollen.

Auffallend ist, dass nur drei Unternehmen überhaupt strategische Überlegungen für das Angebot an dualen Studiengängen nannten, und das mit jeweils unterschiedlichen Zielen: Das Unternehmen der Fahrzeugindustrie nannte als Ziel, mit dualen Studiengängen einem erwarteten Engpass bei Ingenieuren zu begegnen; ein Chemieunternehmen will mit dual Studierenden das Potenzial unterschiedlicher Fä-

(7) Dieser Befürchtung gingen Dobischat, R./Fischell, M./Rosendahl, A. (2008) nach: Auswirkungen der Studienreform durch die Einführung des Bachelorabschlusses auf das Berufsbildungssystem. Eine Problemskizze, edition der HansBöckler-Stiftung (223), Düsseldorf. 
higkeiten im Unternehmen verbessern; das Telekommunikationsunternehmen sieht ganz allgemein die Möglichkeit, besonders leistungsfähige und -bereite Mitarbeiterinnen und Mitarbeiter zu gewinnen.

Einen dahinterstehenden ausbildungsstrategischen Vorteil für die Betriebe zeigt die lerntheoretische Diskussion zum „situierten Lernen“ auf: ${ }^{8}$ Handlungskompetenz kann sich nur entwickeln, wenn Wissen in einen entsprechenden Anwendungskontext integriert wird. Diese Anforderung lässt sich mit dualen Studiengängen schneller erfüllen als über Einarbeitung und betriebliche Sozialisation im Anschluss an wissenschaftsorientierte Hochschulausbildung.

\section{Bildungspolitische Fragestellungen}

Ob duale Studiengänge zukünftig ein betriebswirtschaftlich und gesellschaftlich relevanter Qualifizierungsweg werden, liegt an bildungspolitischen Entscheidungen und am Entwicklungspfad von Facharbeit in den Betrieben; Letzterer hängt nach unserer Einschätzung auch davon ab, inwieweit die Ausbildungsordnungen neue Anforderungen der Arbeitswelt (z. B. die nach Prozess- und Systemkompetenzen) umsetzungsrelevant aufnehmen. Derzeit geben die Freiräume in der rechtlichen Grauzone dieser Angebote den Betrieben die Möglichkeit für eigene neue Qualifizierungsformen. In den Betrieben unserer Fallstudien scheint der Ausbildungsvertrag nach Berufsbildungsgesetz eher eine Notlösung angesichts eines fehlenden Rechtsrahmens zu sein. Dafür sind in den Großbetrieben auch die betriebspolitischen Orientierungen an „bewährten“ und im Rahmen der industriellen Beziehungen konsensfähigen Rechtsformen verantwortlich. In der Vielzahl der (überwiegend kleineren) Betriebe, die sich an praxisintegrierenden dualen Angeboten der Hochschulen beteiligen, dürfte das anders sein: Dort werden Studierende vorwiegend mit Praktikantenstatus im Betrieb integriert. Die Ausbildung erfolgt nach den Studienordnungen der Hochschule, jedoch auf der Grundlage von Einstellungsentscheidungen der Betriebe.

Als ein wichtiges Argument für duale Lernkonzepte gilt die Verbindung von theoretischem und praktischem Lernen. Aber schon in der Ausbildung nach Berufsbildungsgesetz ist die Realisierung dualen Lernens in der Praxis keinesfalls selbstverständlich. In unseren Fallbeispielen hat die betriebliche Praxis keinen systematischen Stellenwert, der sich extra in der Vergabe von Kreditpunkten für den betrieblichen Teil zeigen würde. Es fehlen bisher curriculare Konzepte für duales Lernen in Studiengängen. „Dual“ bedeutet hier nur das Lernen an zwei verschiedenen Lernorten, die weitgehend unabhängig voneinander sind. So entspricht der praktische Teil der Hochschulausbildung eher einem vorgezogenen Traineeprogramm als einem Teil eines im Wortsinn „dualen“ Studiums. Damit wird der oben genannte ausbildungsstrategische Vorteil nicht ausgeschöpft.

Für einen Rechtsrahmen außerhalb des Hochschulrechts ist an eine Regelung im BBiG zu denken. Nur dieses (oder ein vergleichbares Gesetz im Rahmen des Arbeitsrechts) bietet die Möglichkeit einer bundeseinheitlichen Regelung. Im BBiG ließe sich der Lernort Berufsschule durch die Option der Hochschule ergänzen. Dabei wäre allerdings zu klären, wie eine Abstimmung zwischen den Lernorten erreicht werden kann, ohne die verfassungsrechtlich garantierte Autonomie der Hochschulen einzuschränken.

Die Alternative im Hochschulrecht besteht darin, duale Studiengänge mit systematischer Anerkennung betrieblicher Praxisanteile zu konzipieren und zu akkreditieren.

\section{Neue Bildungschancen?}

Die dualen Studienangebote in den untersuchten Betrieben bedeuten nicht grundlegend geänderte Rekrutierungsmuster, etwa als Reaktion auf Anforderungen der Wissensgesellschaft, und sie verdrängen (bisher) auch nicht die klassische Ausbildung nach Berufsbildungsgesetz. Sie sind eher als „Suchbewegungen“ der Unternehmen zu werten, um ihren Bedarf an betriebsnah qualifizierten mittleren Führungskräften sicherzustellen. Das Angebot an qualifizierten Abiturienten und die rechtlichen Rahmenbedingungen in dieser „Grauzone“ ermöglichen ihnen das. Offen ist, ob wie von mancher Seite angenommen - duale Studiengänge einen Beitrag zur Erhöhung der Bildungsbeteiligung und damit zur Erweiterung von Bildungschancen leisten können. ${ }^{\ominus}$ Unsere Fallstudien zeigen jedenfalls, dass durch die betrieblichen Auswahlverfahren und die zeitlich anspruchsvolle Verbindung von Studium, Ausbildung und Arbeit eher neue betriebliche Leistungseliten sozialisiert werden. Deshalb ist die Eröffnung zusätzlicher Bildungschancen zweifelhaft.

Exemplarische Untersuchungen an einer dualen Fachhochschule in Baden-Württemberg geben allerdings Hinweise darauf, dass dort in stärkerem Ausmaß als an Universitäten Studierende mit „weniger günstigem familiären Hintergrund“ studieren. Von daher wird angenommen, dieser Hochschultyp könne eine Brücke darstellen für einen sozialen Aufstieg im betrieblichen Kontext ohne Bruch mit dem Herkunftsmilieu und ohne habituelle Konflikte. Somit könne sich ein neuer ökonomischer Bildungstypus entwickeln, in dem wirtschaftlich-berufliche Orientierung als Zugangsvoraussetzung und zugleich als Merkmal des Studiums gelten. ${ }^{(1)}$

Droht wegen der einzelbetrieblichen Entscheidungen über den Zugang zu dualen Studiengängen eine neue

8 Vgl. Mandl, H./Gerstenmaier, J. (Hrsg.) (2000): Die Kluft zwischen Wissen und Handeln, Göttingen/Bern/Toronto/ Seattle.

(9 So derTenor bei Berthold, C. et al. (vgl. Fußnote 2).

(10 Vgl. Budde, J. (2010): Duales Studium als Aufstiegsprojekt? Bildungswege, Milieulagen und Habitus von Studierenden an Berufsakademien, in: Die Hochschule (1), S. 135-147. 
soziale Exklusion auf dieser Bildungsebene? In den Betrieben unserer Fallstudien scheint das bisher nicht so zu sein, externe Studienabsolventen werden auch weiterhin gerade für höherwertige betriebliche Positionen rekrutiert.

Wir sehen die Problematik derzeit eher im Verhältnis zur klassischen dualen Ausbildung: Auch wenn hier kein Verdrängungseffekt durch dual Studierende zu beobachten ist, ${ }^{\oplus}$ werden durch Ausweitung dualer Studiengänge die Karrierechancen von Absolventen des dualen Systems verringert, weil die dualen Bachelors von den Unternehmen bevorzugt auf solche Positionen gesetzt werden, die bislang durch betriebsinterne Aufstiegsweiterbildung erreichbar waren. Bildungspolitisch scheint es uns von daher sinnvoll, duale Studiengänge berufsbegleitend als Weiterbildung anzubie-

(11) So auch Dobischat et al. (vgl. Fußnote 7).

(12) Vgl. hierzu auch Koch, J./Meerten, E. (2010): Berufsorientierte Weiterbildung in Bachelorstudiengängen realisieren, in: Berufsbildung in Wissenschaft und Praxis (BWP) 39 (2), S. 10-13. ten, und dies vor allem für Fachkräfte ohne schulisch erworbene Studienberechtigung. ${ }^{\text {(1) }}$

\section{AUTOREN}

WINFRIED HEIDEMANN, Dipl.-Soziologe, Dr. phil., seit Juni 2012 im Ruhestand. Er leitete bis dahin das Referat Qualifikation in der Abteilung Mitbestimmungsförderung der Hans-Böckler-Stiftung. Arbeitsschwerpunkte: Institutionenberatung zu Lebenslangem Lernen, europäischer Berufsbildungspolitik und Unternehmensverantwortung.

winfried-heidemann@t-online.de

JOHANNES косн, Dipl.-Pädagoge, leitet das Friedrichsdorfer Büro für Bildungsplanung in Berlin. Arbeitsschwerpunkte: Aktivierende Ausbildungsmethoden, E-Learning und Wissensmanagement.

fb-biplan@t-online.de 